\title{
Below “Optical Diffraction-Limited” Readout in Erasable Optical Disks
}

\author{
Han-Ping D. Shieh, Jia-Reuy Liu, and Wein-Kuen Hwang \\ Institute of Electro-Optic Engineering, National Chiao Tung University, \\ Hsinchu, Taiwan 30010, Rep. Of China
}

Erasable optical storage has not reached adequate capacity of more than 10 Gbyte/disk for vast consumer/multimedia applications because laser spot size focused on the disk media, thus the recording density, is restricted by the law of diffraction which is about 0.6 $\lambda / \mathrm{NA}$, where $\lambda$ and NA are the laser wavelength and numerical aperture of the focusing lens, respectively. Various optical super resolution methods have been proposed in magneto-optical (MO), pre-mastered ROM, and erasable phase change (PC) media to circumvent "diffraction limited", thus, increase recording density. Moreover, most proposed optical super resolutions are also compatible with methods for higher density, including the use of green-blue laser, high NA lens, near-field recording, and advanced signal processing, hence, super resolution provides multiple value in optical storage.

Optical super resolution utilizes disk structures to form a detection aperture much smaller than the readout spot defined by diffraction by masking part of the readout aperture. Magnetic super resolution (MSR) using magnetically coupled of different MO layers has shown to form a detection aperture as small as $0.3 \mathrm{um}$ on the readout layer to retrieve information stored in storage layer. ${ }^{1}$ Moreover, bias magnetic field as low as 100 Oe for magnetic field modulation can also be achieved by properly selected magnetic coupling strength and disk structure as depicted in Fig. 1 to offer added value in MSR detection technique. ${ }^{2}$

In contrast, optical super resolution of detecting "below-diffraction-limited" marks in an erasable PC recording is ascribed to the sharp temperature dependence of refractive index of the recording layers above the melting temperature. The disk is composed of a mask layer, and a recording layer as the active layer; both layers were composed of PC medium GeTeSb with different ratios of contents, thus, different critical cooling rate, as shown in Fig. 2. Three dielectric layers and $\mathrm{Al}$ reflective layer are used to control thermal and optical properties of the disk so that sub-um marks can be recorded and adequate signal contrast can be yielded on the "below-diffraction-limited" marks within readout spot." Fig. 3 depicts reflectioncoefficient profiles of detected and masked area of readout spot in a properly designed PC disk. Optical, thermal designed and thus fabricated disks have shown that 0.4 um marks can be recorded and playback with reasonable CNR using $780 \mathrm{~nm}$ laser and 0.55 NA lens, a factor of 4 improved in recording density. ${ }^{4}$ Optimization of disk structure, materials and readout scheme are being studied to further increase the density, thus, capacity of the erasable PC disks so that DVD-RAM capacity can be enlarged to $20 \mathrm{~GB} /$ disks with current optical system. 


\section{Reference}

1. M. Kaneko, K. Aratani, A. Fukumoto, and S. Miyaoka, Proc. IEEE, 82544 (1994).

2. Wein-Kuen Hwang and Han-Ping D. Shieh, J. Appl. Phys., 81 (6), 2745(1997).

3. Jia-Reuy Liu and Han-Ping D. Shieh, Jpn. J. Appl. Phys. 37(5A), L516(1998).

4. Jia-Reuy Liu, Nai-Yuan Tang, and Han-Ping D. Shieh, Appl. Opt., Accepted for publication, July. 1998.

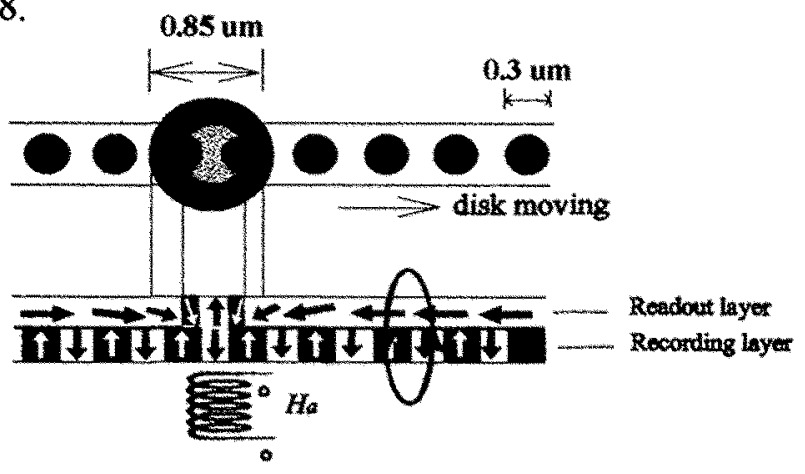

Fig. 1 MSR readout scheme with low bias field in MO

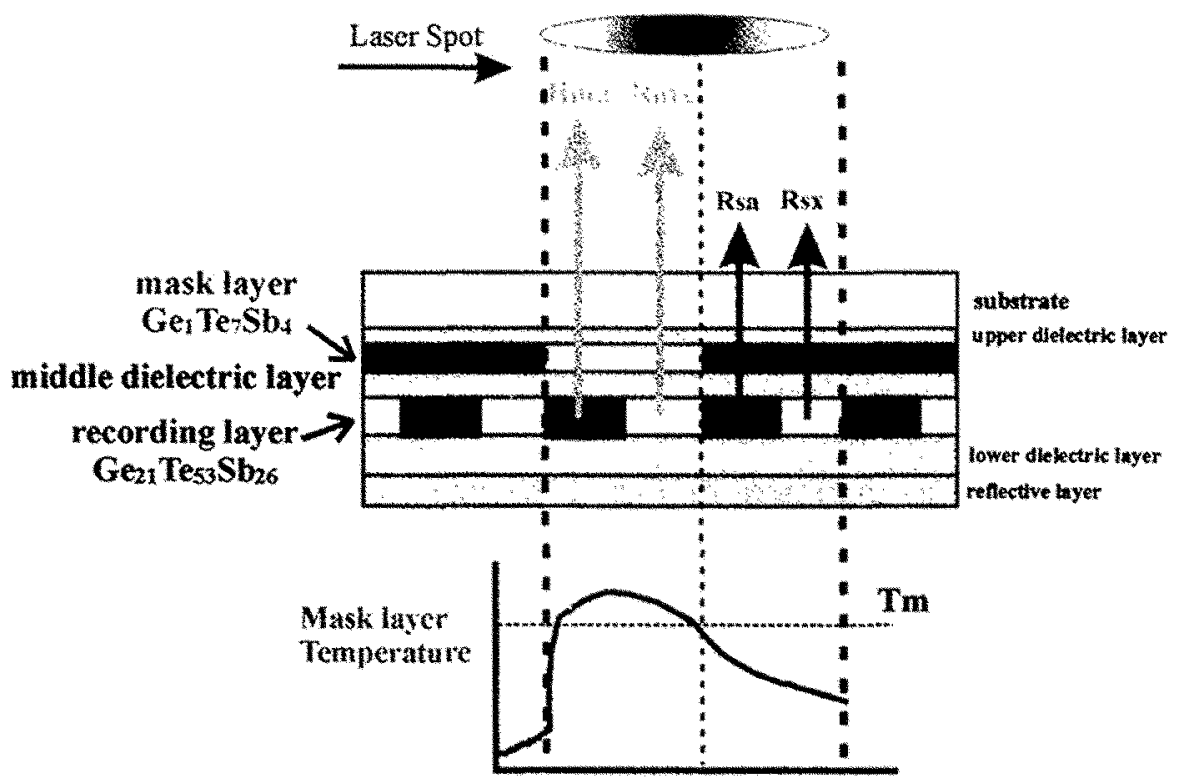

Fig. 2 Optical super resolution in erasable phase disk and temperature profile during reading

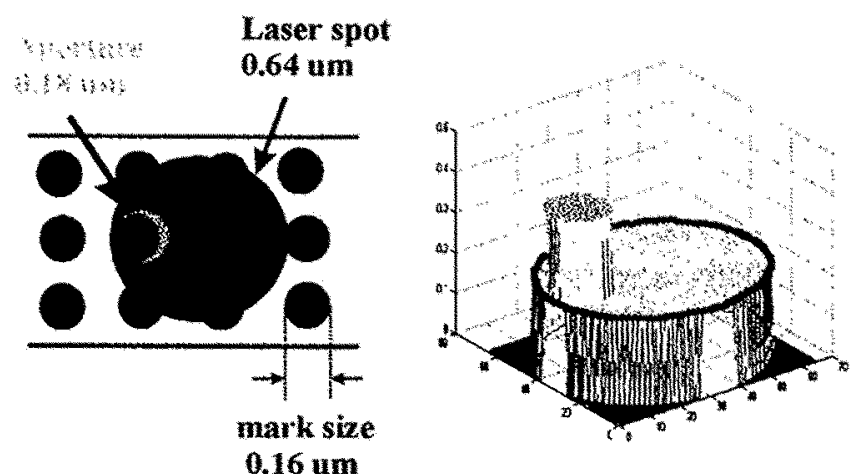

Fig. 3 Reflection-coefficent profile of optical super resolution on 0.16 um marks with 0.64 um laser spot 